\title{
Electronically monitored medication adherence predicts hospitalization in heart failure patients
}

\author{
This article was published in the following Dove Press journal: \\ Patient Preference and Adherence \\ 4 December 2013 \\ Number of times this article has been viewed
}

\section{Barbara Riegel ${ }^{1,2}$ \\ George J Knafl ${ }^{3}$}

'University of Pennsylvania School of Nursing, ${ }^{2}$ University of Pennsylvania Leonard Davis Institute, Philadelphia, PA, USA; ${ }^{3}$ University of North Carolina School of Nursing,

Chapel Hill, NC, USA
Correspondence: Barbara Riegel University of Pennsylvania School of Nursing, 418 Curie Boulevard, Philadelphia, PA 19104-4217, USA Tel +l 2158989927

Fax + I 2402827707

Email briegel@nursing.upenn.edu
Background: Hospitalization contributes enormously to health care costs associated with heart failure. Many investigators have attempted to predict hospitalization in these patients. None of these models has been highly effective in prediction, suggesting that important risk factors remain unidentified.

Purpose: To assess prospectively collected medication adherence, objectively measured by the Medication Event Monitoring System, as a predictor of hospitalization in heart failure patients.

Materials and methods: We used recently developed adaptive modeling methods to describe patterns of medication adherence in a sample of heart failure patients, and tested the hypothesis that poor medication adherence as determined by adaptive methods was a significant predictor of hospitalization within 6 months.

Results: Medication adherence was the best predictor of hospitalization. Besides two dimensions of poor adherence (adherence pattern type and low percentage of prescribed doses taken), four other single factors predicted hospitalization: low hemoglobin, depressed ejection fraction, New York Heart Association class IV, and 12 or more medications taken daily. Seven interactions increased the predictive capability of the model: 1) pattern of poor adherence type and lower score on the Letter-Number Sequencing test, a measure of short-term memory; 2) higher number of comorbid conditions and higher number of daily medications; 3) higher blood urea nitrogen and lower percentage of prescribed doses taken; 4) lower hemoglobin and much worse perceived health compared to last year; 5) older age and lower score on the Telephone Interview of Cognitive Status; 6) higher body mass index and lower hemoglobin; and 7) lower ejection fraction and higher fatigue. Patients with none of these seven interactions had a hospitalization rate of $9.7 \%$. For those with five of these interaction risk factors, $100 \%$ were hospitalized. The $C$-index (the area under the receiver-operating characteristics [ROC] curve) for the model based on the seven interactions was 0.83 , indicating excellent discrimination.

Conclusion: Medication adherence adds important new information to the list of variables previously shown to predict hospitalization in adults with heart failure.

Keywords: heart failure, outcomes, hospitalization, patient compliance, medication adherence, self-care

\section{Introduction}

Heart failure (HF) is the most prevalent and fastest-growing cardiovascular disease worldwide. ${ }^{1}$ In the US among those 80 years of age and older, almost $12 \%$ of men and women have $\mathrm{HF}$, and the prevalence is projected to increase by $25 \%$ by $2030 .{ }^{1}$ Outcomes associated with HF include poor quality of life, high mortality, and frequent hospitalizations that contribute enormously to health care costs. ${ }^{1}$ 
In 2010, the Patient Protection and Affordable Care Act was enacted to address the rising costs of health care in the US. ${ }^{2}$ In 2012, value-based purchasing was introduced, and hospitals began being financially spurred to produce quality outcomes while reducing inappropriate spending. Specifically, the Centers for Medicare and Medicaid Services Readmissions Reduction Program financially penalizes health care systems when the 30-day all-cause readmission rates for Medicare patients are higher than expected in particular diagnostic categories. HF is one of the targeted diagnoses, so efforts to identify modifiable factors predicting HF hospitalizations are increasingly important.

Numerous investigators have attempted to predict hospitalization in adults with HF. In a recent review, Giamouzis et $\mathrm{al}^{2}$ summarized risk factors for hospitalization as sociodemographic, clinical, blood and other test results, comorbidity burden, and cardiovascular, noncardiovascular, quality of life, psychosocial, and disease-management factors. ${ }^{2}$ Others have attempted to synthesize these results by developing models that can be used to predict hospitalization risk. ${ }^{3-9}$ None of these models has been highly effective in prediction. The $C$-index (the area under the receiver-operating characteristics [ROC] curve) in the various studies ranged from 0.57 to 0.74 , suggesting that important risk factors remain unidentified. The purpose of this study was to assess medication adherence as a predictor of hospitalization in adults with HF in conjunction with other possible predictors.

Poor medication adherence is ubiquitous in chronically ill patient populations. ${ }^{10}$ In a review of medication adherence in $\mathrm{HF}, \mathrm{Wu}$ et $\mathrm{al}^{11}$ noted that most investigators have found nonadherence rates of $40 \%-60 \%$ of HF patients. Prior studies have demonstrated that disease-management interventions emphasizing evidence-based medications can decrease hospitalization rates, ${ }^{12}$ but few studies have addressed the risk of hospitalization associated with medication nonadherence in adults with HF. The few studies that have been done suggest that hospitalization rates are higher when medication adherence is poor. ${ }^{13-16}$ These studies are limited, however, to retrospective analyses of such electronic data as pharmacy records.

In this study, we describe patterns of prospectively collected, objectively measured medication adherence in a sample of HF patients and test the hypothesis that medication adherence is a significant predictor of hospitalization within 6 months. This approach addresses several important gaps in the literature. First, collecting the data prospectively negates the need for decision rules regarding the coding of electronic data. Second, medication adherence was measured objectively with the Medication Event Monitoring System $\left(\mathrm{MEMS}^{\circledR}\right)$ (Aardex; MW Healthcare, Richmond, VA, USA), which is more accurate than self-reported medicationadherence data. Third, we used a statistical approach ${ }^{17,18}$ that allowed us to utilize more fully these medication-adherence data to characterize patients' adherence patterns and to identify adherence types with similar patterns, rather than the usual approach of classifying adherence using an arbitrarily devised cutoff (usually $>80 \%$ ). ${ }^{19}$ Fourth, while these methods have been used before to model MEMS adherence data for human immunodeficiency virus-positive patients ${ }^{17,20}$ and for African-American patients with hypertension, ${ }^{21}$ they have not been used before with adherence data from HF patients.

\section{Materials and methods}

This was a planned analysis of longitudinal data from a prospective cohort study of a consecutive sample of 280 adults with HF. Subjects were enrolled from three outpatient settings in the northeastern US. Institutional review board approval was obtained at each site, and all participants gave informed consent. Data were collected between 2007 and 2009. A detailed description of study methods has been published previously. ${ }^{22}$

Patients with a confirmed diagnosis of chronic HF were enrolled, regardless of etiology or ejection fraction. Inclusion criteria addressed the ability to participate (eg, vision, hearing, English literacy, and cognition). ${ }^{15}$ Cognition was screened using the Telephone Interview of Cognitive Status (TICS). ${ }^{23}$ Exclusion criteria were major depressive illness, dementia, renal failure requiring dialysis, or a recent history of serious drug or alcohol abuse.

Participants were followed for 6 months, with study visits at baseline, 3 , and 6 months. Data on self-care and cognition were collected at each study visit. Most data were collected during home visits by research assistants. Nurses abstracted clinical information from medical records. For this analysis, we tested a wide range of demographic, social support, clinical, self-care, symptom, and cognition variables, so as to add to the knowledge of significant predictors previously identified by others. ${ }^{2}$

The outcome variable of hospitalization was measured using the electronic medical record at each participating institution. In addition, participants were telephoned monthly and asked about hospitalizations (and also emergency department visits). Medical records were obtained if the visit took place at a hospital outside of the home institution. In this analysis, all hospitalizations were analyzed, 
regardless of cause (not including emergency department visits).

\section{Measures}

MEMS was used to measure medication adherence objectively. Our methods for collecting MEMS data are described in detail elsewhere, ${ }^{24}$ the MEMS data were used differently in that study. In brief, using a microprocessor in the cap, the $\mathrm{MEMS}^{\circledR} 6$ device measures openings that are presumed to be for the purpose of removing a pill to take it orally. MEMS data were collected on one medication scheduled to be taken at fixed times. MEMS data were collected over a 6-month interval and downloaded at 3 and 6 months. Deviations in use, such as accidental openings, were noted in study diaries and used to correct the time-stamped medication events stored in the device before analysis. In this study, we considered three aspects of adherence based on MEMS data: adherence-pattern types, consistency of adherence patterns with the prescribed rate, and the percentage of prescribed doses taken (PDT). The medication controlled by MEMS did not change for any of the patients during the study. For one patient, the prescribed rate for the medication controlled by MEMS changed during the study. The adherence pattern for this patient was adjusted to account for this change.

Demographic variables, such as age, race, and sex were collected by self-report. In addition to measuring education as the number of years of formal schooling, the American National Adult Reading Test (ANART) was administered as a test of premorbid intellect.

Social support was measured in multiple ways. Marital status was self-reported, as was living alone (yes/no) and the overall quality of the support received defined as "emotional support, information, material help, errands, etc," with responses ranging from 1 (poor) to 4 (very good). In addition, support from family, friends, and significant others was assessed using the Multidimensional Scale of Perceived Social Support (MSPSS). ${ }^{25}$ Responses range from 1 (very strongly disagree) to 7 (very strongly agree), with higher scores indicating higher perceptions of support.

Clinical variables included health-related factors, such as exercise in the last week, which was assessed by self-report with responses of 1 (none) to 4 (more than 3 hours). Body mass index (BMI) was calculated from self-reported height and measured body weight. Variables abstracted from the medical record included months since diagnosed with HF, most recent blood urea nitrogen (BUN), creatinine, hemoglobin ( $\mathrm{Hgb})$, serum sodium, and ejection fraction. The Charlson Comorbidity Index ${ }^{26}$ was completed based on chart review.
Any history of sleep apnea, atrial fibrillation, hypertension, diabetes, cerebral disease, renal disease, anemia, or pulmonary hypertension was noted. Pulse and systolic and diastolic blood pressure were measured during the enrollment visit. Treatment quality was rated separately for systolic and diastolic HF, using those treatments advocated in HF clinical guidelines (eg, angiotensin-converting enzyme-inhibitor use, beta-blocker use) in place at the time. ${ }^{27}$

Self-care was measured with the Self-Care of HF Index (SCHFI), which assesses self-care maintenance, management, and confidence. ${ }^{28}$ Each scale is standardized to a score of 100 , with higher scores indicating better self-care. Knowledge of HF was assessed using the Dutch HF Knowledge Scale ${ }^{29}$ with 15 dichotomously scored items; higher scores indicate higher knowledge of HF treatment, HF symptoms, and symptom recognition. The number of medications taken daily was calculated based on a review of medication containers by research assistants during home visits.

Symptoms measured by self-report included trouble breathing or ankle swelling in the past month (yes/no), general health perceptions, rated 1 (excellent) to 5 (poor), and health perception compared to 1 year prior, rated 1 (much better now than 1 year ago) to 5 (much worse now than 1 year ago). Information on functional class was gathered during the home visit using a standardized interview. A single cardiologist scored New York Heart Association (NYHA) class in every subject. Fatigue was measured using two items from the Kansas City Cardiomyopathy Questionnaire. ${ }^{30}$ Each item is scored 1 to 7; scores were reversed so that higher scores indicated more fatigue. Sleepiness was assessed with the Stanford Sleepiness Scale ${ }^{31}$ and the Epworth Sleepiness Scale (ESS). ${ }^{32}$ ESS respondents rate the likelihood of falling asleep in eight soporific situations on a scale ranging from 0 (never dozing) to 3 (high chance of dozing). ESS scores are summed, with higher scores indicating higher sleepiness or categorized as sleepy $(\geq 11)$ or not sleepy $(<11)$. Sleep quality was assessed with the Pittsburgh Sleep Quality Index. ${ }^{33}$ A global score (0-21 points) is obtained by summing the scale domain scores. Higher scores indicate poorer sleep quality; a score $>5$ is categorized as poor sleep. Depression was measured using the Patient Health Questionnaire. ${ }^{34}$ Responses range from 0 (not at all) to 3 (nearly every day), with higher scores indicating more depression.

A neuropsychological test battery was administered to measure simple attention (Psychomotor Vigilance Task [PVT]), complex attention (Trail Making Test B), processing speed (Digit Symbol Substitution Test, Trail Making Test A), working memory (Probed Recall Memory task), 
and short-term memory (Letter-Number Sequencing [LNS] test). ${ }^{35}$ Transformed lapses $>4.69$ on the PVT were scored as abnormal. ${ }^{36}$ Scores on each of the other tests were classified based on age-based norms. In addition, the number of tests scored as abnormal was used as a summary measure of cognitive status. Anyone scoring $>1.5$ standard deviations on two or more of the cognition tests was scored as having cognitive decline. Efforts made to compensate for perceived memory issues were measured and summarized in the Compensatory Activities Scale.

\section{Data analysis}

Other than standard analyses (eg, descriptive statistics), analyses were conducted using the adaptive methods of Knafl et al. ${ }^{17}$ These methods were used to characterize adherence patterns for individual patients over their 6 months in the study, classify these patterns into adherence types, and identify dichotomous risk factors, individually and in combination, for hospitalization.

\section{Likelihood cross-validation}

Adaptive methods use a $K$-fold likelihood cross-validation (LCV) approach for evaluating and comparing alternative models. Observations (eg, daily MEMS use) are randomly partitioned into $K$ disjoint subsets called folds. For a given model, the likelihood for the data in each fold is computed from model parameter values estimated using the data in the other folds. These deleted fold likelihoods are normalized by the sample size and multiplied up into the LCV score for the model. Larger LCV scores indicate better models for the data under analysis. LCV scores can be used for model selection in any analysis context where parameter estimation is based on maximizing a likelihood or any likelihood-like function (eg, a quasi-likelihood). All analyses used 10-fold LCV scores.

A larger LCV score does not necessarily mean the model is preferable. If the smaller LCV score is not too much smaller and the associated model has a simpler structure (eg, using an untransformed predictor compared to using the log transform of that predictor), then this model is preferable as a parsimonious, competitive alternative. LCV-ratio tests, analogous to likelihood-ratio tests, can be used to assess whether a smaller LCV score is substantially (significantly or distinctly) smaller or not. These are $\chi^{2}$-based tests and are expressed in terms of a cutoff for a substantial percent decrease in the LCV score. The cutoff changes with the sample size (for the formula, see [6] of Knafl et $\mathrm{al}^{17}$ ).

\section{Individual adherence patterns}

The study-participation period for each patient was subdivided into equally sized intervals of length at least 3 days with no more than 100 intervals. Counts of MEMS cap openings and opening rates per day per dose were computed for each interval. These counts and rates were modeled using Poisson regression models (as is appropriate for such data). Both mean adherence and adherence variability based on dispersion parameters ${ }^{37}$ were modeled as possibly nonlinear functions of time during study participation. LCV scores were based on extended quasi-likelihoods.

The predictors for the Poisson regression models were power transforms of time with possibly fractional (ie, noninteger) powers, and so these are called fractional polynomial models. ${ }^{38}$ An adaptive process was used to identify an effective set of such power transforms. The adaptive process starts from the constant model and systematically expands the model, adding in power transforms of time to either the mean adherence or dispersion component of the model, continuing as long as the LCV score does not decrease by too much (as determined by an expansion-stopping tolerance parameter). The expanded model is then contracted by removing transforms of time or possibly the intercept term as long as the LCV score does not decrease by too much (as determined by a contraction-stopping tolerance parameter). The transforms of the contracted model determine the adherence pattern underlying the data for how both mean adherence and adherence variability change over time (examples are given in Figure 1, as described in the "Results" section).

Since the prescribed rates were not the same for all patients (ranging from one to three doses per day), adherence patterns were normalized by the prescribed number of doses to be comparable. The ideal adherence pattern is then the one with constant mean adherence equal to 1 and constant adherence variability equal to 0 . A summary measure of how consistent the observed adherence pattern is with this ideal pattern is calculated for each patient as a percentage, and so is called percentage consistency.

\section{Adherence types}

Mean adherence and adherence variability were estimated at 20 proportionally spaced times within each patient's study participation period $(5 \%, 10 \%, \ldots 100 \%)$. The 40 vectors formed from these two types of estimates were clustered into adherence types, ie, sets of patients with similar mean adherence and adherence variability over time. A wide variety of clustering procedures with varying numbers of clusters were considered. ${ }^{17}$ These were compared using LCV 

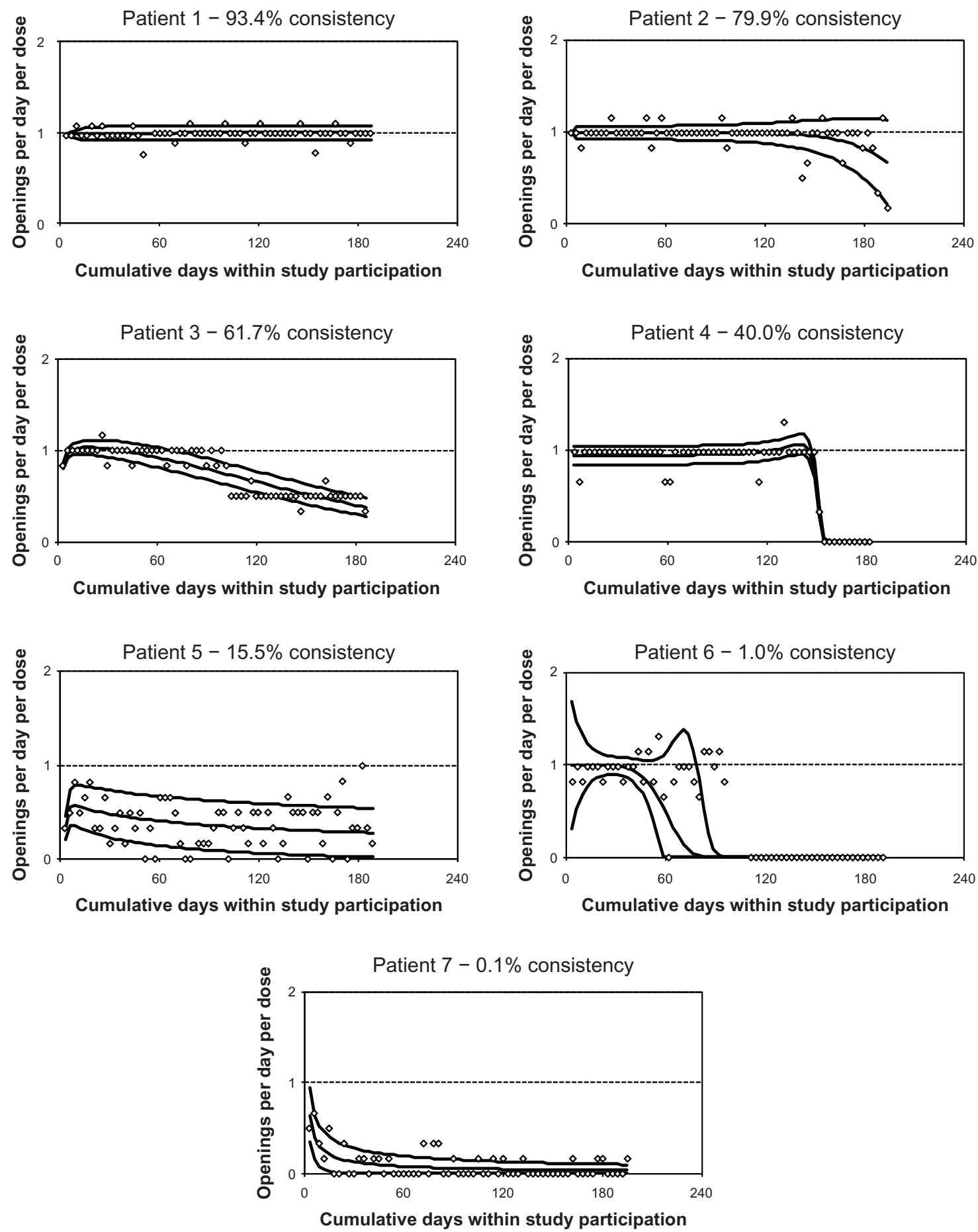

Figure I Sample adherence patterns.

Notes: The raw data are plotted as diamonds. The middle curve is the estimated mean adherence over time while the other curves are unit error bands, \pm I estimated standard deviation around mean adherence.

scores based on likelihoods for multivariate normal-mixture models (as is appropriate for cluster analyses). The selected clustering alternative was the one generating the best score among those with at least $5 \%$ of the patients in each cluster (to avoid sparse clusters). Plots of averages of mean adherence and adherence variability (ie, the centroids) were used in interpreting the clusters (see Figure 2, as described in the "Results" section).

\section{Identifying risk factors for hospitalization}

Potential dichotomous risk factors were identified for the dichotomous hospitalization-outcome variable. 


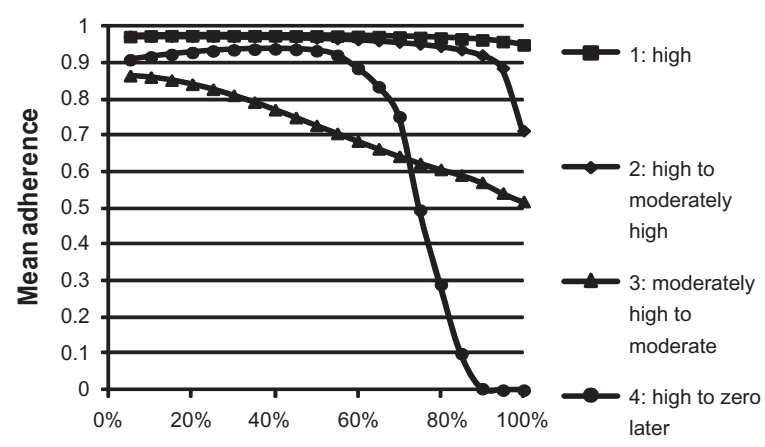

Percentage of study participation

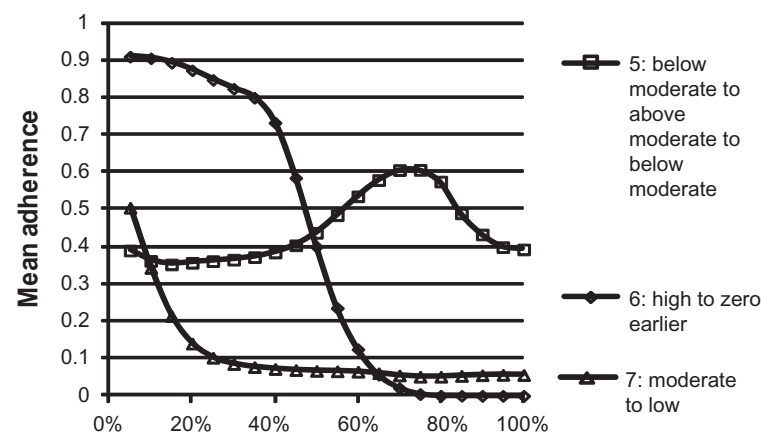

Percentage of study participation

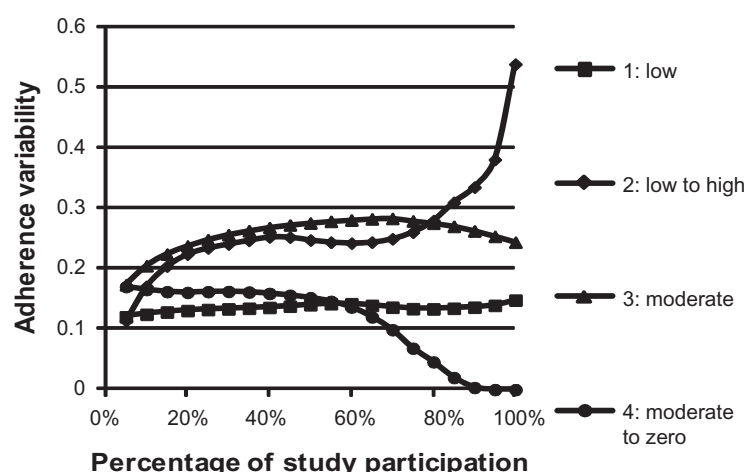

Percentage of study participation

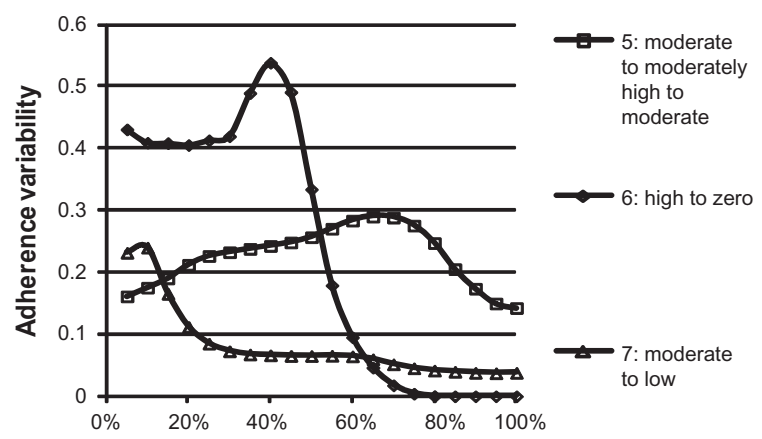

Percentage of study participation

Figure 2 Plots of average mean adherence and of average adherence variability for patients in adherence types.

Most categorical predictors with more than two values were reduced to dichotomous predictors expected to be risk factors. For example, income level was reduced to not enough as the risk factor versus enough or more than enough. Otherwise, categorical predictors were represented by multiple dichotomous risk factors. For example, employment status was represented by being retired versus not and being unemployed or disabled versus not. For each continuous and ordinal predictor, its observed values were adaptively grouped into two subsets of contiguous values. For each observed value of the predictor (or a rounded value to reduce the number of decimal digits) as a cutoff, LCV scores were computed for the model based on the indicator for having values above or below the cutoff. When a variable had missing values, these were conservatively grouped with the non-risk-factor values so that the effect of the associated risk factor held only for patients with nonmissing values for the underlying variable. The cutoff generating the best LCV score was chosen and the risk factor defined in terms of values either above or below the cutoff, depending on which of these choices had a positive effect on the outcome variable, ie, with odds ratio $>1$. To avoid sparse cases, only those cutoffs were considered for which at least $5 \%$ of the observations fell both above and below the cutoff. When a standard cutoff existed for a predictor (eg, PVT >4.69), that categorization was considered as well.

To be consistent with the handling of other predictors, adherence type was adaptively reduced to a dichotomous risk factor of poor-adherence types versus better-adherence types. Models of hospitalization were used for this purpose. The decision regarding which adherence types to assign to the two alternatives was made adaptively using LCV scores. In contrast to the handling of values for continuous and ordinal predictors, the alternate adherence types were not considered to be ordered.

Data for patients with at least some MEMS data were used to model hospitalization in terms of adherence and other available risk factors, and so data for patients with no MEMS data were missing for these analyses. Only outcome means were adaptively modeled and not outcome dispersions, using unit dispersions for all models (as for standard logistic regression modeling). Bivariate models were generated for all potential risk factors. Then, a multiple risk-factor model was generated considering only the risk factors with a significant $(P<0.05)$ bivariate effect. The adaptive modeling process, as used for generating individual patient adherence patterns in terms of the single predictor of time, was used for this purpose. This multiple risk-factor model provides a 
parsimonious depiction of the combination of risk factors that affect hospitalization. Next, interactions between the pooradherence type risk factor with each of the other available risk factors were considered by starting with the model based on these two risk factors and their interaction and adaptively reducing that model to identify cases when an interaction term remained in the model. A multiple risk-factor model was generated considering these identified interaction effects along with the noninteraction risk factors with significant individual effects. Finally, an adaptive model was generated considering this same set of risk factors as well as possible interactions between any two of them to obtain a fuller assessment of interactions between risk factors.

\section{Results}

\section{Sample}

A total of $280 \mathrm{HF}$ patients participated in the study. Summary statistics are presented in Tables 1-6 for available variables within the six categories described earlier. For example, patients were primarily male $(64.3 \%)$, white $(62.5 \%)$, and retired (43.9\%). Ages ranged from 24 to 89 years with a mean of 62.0 (standard deviation $=12.5$ ) years, while education ranged from 8 to 29 years with mean of 13.9 (standard deviation $=2.9$ ) years. Over the course of the 6-month study, $103(36.8 \%)$ of the patients were hospitalized.

\section{Individual patient adherence patterns}

MEMS adherence data were available for 218 (77.9\%) patients. Of these patients, $79(36.2 \%)$ were hospitalized

Table I Summary statistics for available demographic variables

\begin{tabular}{|c|c|c|c|}
\hline Variable & Observed range & n (\%) ${ }^{a}$ & $\begin{array}{l}\text { Mean } \\
\text { (SD) }\end{array}$ \\
\hline Employment & Retired & $123(43.9)$ & \\
\hline \multirow[t]{2}{*}{ status } & Unemployed or disabled & $79(28.2)$ & \\
\hline & Employed (full or part time) & $78(27.9)$ & \\
\hline \multirow[t]{2}{*}{ Sex } & Male & $180(64.3)$ & \\
\hline & Female & $100(35.7)$ & \\
\hline \multirow[t]{2}{*}{ Income } & Do not have enough & $45(16.1)$ & \\
\hline & $\begin{array}{l}\text { Have enough or more } \\
\text { than enough }\end{array}$ & $235(83.9)$ & \\
\hline \multirow[t]{2}{*}{ Insurance } & Government or none & $155(55.4)$ & \\
\hline & Commercial or HMO & $125(44.6)$ & \\
\hline \multirow[t]{2}{*}{ Race } & Non-white & $105(37.5)$ & \\
\hline & White & $175(62.5)$ & \\
\hline Age, years & $24-89$ & & $62.0(12.5)$ \\
\hline ANART score & $0-49$ & & $29.8(I 1.8)$ \\
\hline $\begin{array}{l}\text { Years of } \\
\text { education }\end{array}$ & $8-29$ & & $13.9(2.9)$ \\
\hline
\end{tabular}

Note: a Out of 280 patients.

Abbreviations: SD, standard deviation; $\mathrm{HMO}$, health maintenance organization; ANART, American National Adult Reading Test.
Table 2 Summary statistics for available social support variables

\begin{tabular}{|c|c|c|c|}
\hline Variable & Observed range & $n(\%)^{a}$ & $\begin{array}{l}\text { Mean } \\
\text { (SD) }\end{array}$ \\
\hline \multirow[t]{2}{*}{ Living alone } & Yes & $59(21.1)$ & \\
\hline & No & 221 (78.9) & \\
\hline \multirow[t]{2}{*}{ Marital status } & $\begin{array}{l}\text { Single, divorced, } \\
\text { separated, or widowed }\end{array}$ & $|2|(43.2)$ & \\
\hline & Married or partnered & $159(56.8)$ & \\
\hline Quality of & Satisfactory to good & $105(37.5)$ & \\
\hline support & Very good & $175(62.5)$ & \\
\hline MSPSS score & $14-84$ & & $72.6(11.5)$ \\
\hline
\end{tabular}

Note: aOut of 280 patients.

Abbreviations: SD, standard deviation; MSPSS, Multidimensional Scale of Perceived Social Support.

Table 3 Summary statistics for available clinical variables

\begin{tabular}{|c|c|c|c|}
\hline Variable & $\begin{array}{l}\text { Observed } \\
\text { range }\end{array}$ & $n(\%)^{a}$ & $\begin{array}{l}\text { Mean } \\
\text { (SD) }\end{array}$ \\
\hline \multirow[t]{2}{*}{ Exercise } & None & $57(20.4)$ & \\
\hline & Some & $223(79.6)$ & \\
\hline $\begin{array}{l}\text { Adherence to treatment } \\
\text { guidelines }\end{array}$ & $0.00-1.00$ & & $0.8(0.2)$ \\
\hline Body mass index & $15.0-67.0$ & & $30.9(7.9)$ \\
\hline Blood urea nitrogen & $6-97$ & & $25.4(15.1)$ \\
\hline Charlson total & $I-11$ & & $2.8(1.7)$ \\
\hline Comorbidities & $0-9$ & & $3.1(2.1)$ \\
\hline Creatinine & $0.5-3.4$ & & $1.3(0.6)$ \\
\hline Diastolic blood pressure & $45-103$ & & $69.0(11.2)$ \\
\hline Ejection fraction & $5-80$ & & $35.4(17.0)$ \\
\hline Hemoglobin & $7.9-18.4$ & & $13.0(1.8)$ \\
\hline $\begin{array}{l}\text { Months since heart } \\
\text { failure diagnosis }\end{array}$ & $0-508$ & & 73.4 (7I.I) \\
\hline Pulse & $42-100$ & & $69.7(11.4)$ \\
\hline Serum sodium & $|3|-\mid 46$ & & | $39.0(4.0)$ \\
\hline Systolic blood pressure & $80-176$ & & II6.| (18.4) \\
\hline
\end{tabular}

Note: a Out of 280 patients.

Abbreviation: SD, standard deviation.

Table 4 Summary statistics for available self-care variables

\begin{tabular}{llll}
\hline Variable & $\begin{array}{l}\text { Observed } \\
\text { range }\end{array}$ & $\mathbf{n}(\%)$ & $\begin{array}{l}\text { Mean } \\
\text { (SD) }\end{array}$ \\
\hline MEMS data available $^{\mathrm{a}}$ & No & $62(22.1)$ & \\
& Yes & $218(77.9)$ & \\
Adherence type $^{\mathrm{b}}$ & Poor & $63(28.9)$ & \\
& Better & $155(77.5)$ & \\
Prescribed rate for medication & $2-3$ & $133(61.0)$ & \\
controlled by MEMS & $\mathrm{I}$ & $85(39.0)$ & \\
DHFKS score & $7-15$ & & $11.7(1.7)$ \\
SCHFI self-care confidence & $42-100$ & & $75.8(14.4)$ \\
SCHFI self-care maintenance & $32-92$ & & $66.8(11.9)$ \\
SCHFI self-care management & $29-100$ & & $67.4(18.7)$ \\
Total medications & $1-25$ & & $9.9(4.0)$ \\
Consistency, \% & $0.1-93.9$ & & $47.6(34.8)$ \\
Prescribed doses taken, \% & $5.8-100$ & & $71.1(25.9)$ \\
\hline
\end{tabular}

Notes: aOut of 280 patients; bout of 218 patients with available MEMS data. Abbreviations: SD, standard deviation; DHFKS, Dutch Heart Failure Knowledge Scale; MEMS, Medication Event Monitoring System; SCHFI, Self-Care of Heart Failure Index. 
Table 5 Summary statistics for available symptom variables

\begin{tabular}{llll}
\hline Variable & Observed range & $\mathbf{n}(\%)^{\mathbf{a}}$ & Mean (SD) \\
\hline $\begin{array}{l}\text { General health } \\
\text { perception }\end{array}$ & Poor & $37(13.2)$ & \\
Health compared to & Fair to excellent & $243(86.8)$ & \\
a year ago & than worse now & $24(8.6)$ & \\
& Not much worse & $256(91.4)$ & \\
& now than a year ago & & \\
Trouble breathing or & Yes & I25 (44.6) & \\
ankle swelling within & No & I55 (55.4) & \\
past month & & & \\
NYHA class & IV & $230(82.1)$ & \\
& I-III & & $6.5(3.2)$ \\
Fatigue & $2-13$ & & $2.3(1.2)$ \\
SSS score & I-6 & & $7.0(4.6)$ \\
ESS score & $0-23$ & & $4.2(4.1)$ \\
PSQI global score & $0-19$ & & \\
PHQ total & $0-18$ & &
\end{tabular}

Note: a Out of 280 patients.

Abbreviations: SD, standard deviation; ESS, Epworth Sleepiness Scale; NYHA, New York Heart Association; PHQ, Patient Health Questionnaire; PSQI, Pittsburgh Sleep Quality Index; SSS, Stanford Sleepiness Scale.

during the study. Hospitalization rates were not significantly different for patients with no MEMS data versus some MEMS data $\left(\chi^{2}[1]=0.13, P=0.722\right)$.

Figure 1 contains plots of adherence patterns for seven exemplar patients. The y-axis of these plots is openings per day per dose with an ideal value of 1 , while the $\mathrm{x}$-axis is cumulative days of study participation. The raw data are plotted as diamonds. The middle curve is the estimated mean adherence over time, while the other curves are unit error bands, \pm 1 estimated standard deviation (as extended to account for nonlinear dispersion) around mean adherence.

Patient 1 had mean adherence close to the prescribed rate with small variability, which was $93.4 \%$ consistent with

Table 6 Summary statistics for available cognition variables

\begin{tabular}{lll}
\hline Variable & Observed range & Mean (SD) \\
\hline CAS score & $0-23$ & $9.2(4.4)$ \\
DSST score & $11-96$ & $53.4(17.5)$ \\
LNS score & $1-20$ & $8.7(3.5)$ \\
PMR score & $0-4$ & $2.0(I .2)$ \\
PVT lapses & $0-79$ & $8.5(I 3.1)$ \\
TICS score & $26-40$ & $33.6(3.1)$ \\
TMTA score & $14-120$ & $43.8(22.3)$ \\
TMTB score & $8-300$ & $111.2(59.1)$ \\
Dimensions cognitively impaired & $0-5$ & $1.7(I .0)$ \\
\hline
\end{tabular}

Abbreviations: SD, standard deviation; CAS, Compensatory Activities Score; DSST, Digit Symbol Substitution Test; LNS, Letter-Number Sequencing; PMR, Probed Memory Recall; PVT, Psychomotor Vigilance Task; TICS, Telephone Interview for Cognitive Status; TMTA, Trail Making Test A; TMTB, Trail Making Test $\mathrm{B}$. adherence at the prescribed rate. Patient 2's mean adherence was close to the prescribed rate, with small variability up to about 120 days of study participation. After that, mean adherence decreased somewhat with increased variability for $79.9 \%$ consistency with the prescribed rate. Patient 3 had deteriorating mean adherence from about the prescribed rate to around half the prescribed rate, with low variability and $61.7 \%$ consistency. Patient 4 's mean adherence was close to the prescribed rate, with small variability up to about 150 days of study participation, after which adherence dropped to essentially zero for $40.0 \%$ consistency. Patient 5 had relatively consistent mean adherence at around half the prescribed rate, with moderate variability and $15.5 \%$ consistency. Patient 6's mean adherence started at about the prescribed rate and decreased to zero by about 90 days of study participation, with variability decreasing for a while and then increasing. After that, adherence remained at zero. This pattern was only $1.0 \%$ consistent with prescribed adherence. In contrast, the PDT for this patient was $48.9 \%$, suggesting that the patient was adherent at around half the prescribed rate. The plot indicates that patient 6 was adherent around the prescribed rate for about half the time and zero adherent the rest of the time, resulting in a percentage PDT close to $50 \%$, but never around $50 \%$ at any time. In cases like this, percentage PDT provides a misleading assessment of adherence, since it is based on an assumption of constant adherence over time. Patient 7 had mean adherence starting around half the prescribed rate and deteriorating quickly to nearly zero, with low variability and $0.1 \%$ consistency.

\section{Adherence types}

Cluster analysis identified seven adherence types. Figure 2 contains plots of averages of mean adherence and of adherence variability for patients in the seven clusters. These plots were used to generate the interpretations of the adherence types given in Table 7. The clusters were numbered so that averages of percentage consistency and percentage PDT decreased. Consequently, cluster 1 corresponded to the best adherence type, with mean adherence consistently very close to the prescribed rate and adherence variability consistently low. Cluster 7 corresponded to the worst adherence type, with mean adherence starting at about half the prescribed rate and moderate adherence variability, then deteriorating quickly to low, not too much above zero mean adherence and adherence variability. The adherence patterns of patients $1-7$ were allocated to clusters $1-7$, respectively. The individual patterns of Figure 1 are only similar to the 
Table 7 Description of adherence types

\begin{tabular}{|c|c|c|c|c|c|c|}
\hline \multirow[t]{2}{*}{ Cluster } & \multirow[t]{2}{*}{$\mathbf{n}$} & \multirow[t]{2}{*}{$\%$} & \multicolumn{2}{|l|}{ Adherence types } & \multirow{2}{*}{$\begin{array}{l}\text { Average \% } \\
\text { consistency }\end{array}$} & \multirow{2}{*}{$\begin{array}{l}\text { Average } \% \text { prescribed } \\
\text { doses taken }\end{array}$} \\
\hline & & & Mean adherence & Adherence variability & & \\
\hline I & 72 & 33.0 & High & Low & 84.8 & 96.0 \\
\hline 2 & 30 & 13.8 & High to moderately high & Low to high & 64.9 & 88.2 \\
\hline 3 & 24 & 11.0 & Moderately high to moderate & Moderate & 49.6 & 71.7 \\
\hline 4 & 18 & 8.3 & High to zero later & Moderate to zero & 30.3 & 70.1 \\
\hline 5 & 29 & 13.3 & $\begin{array}{l}\text { Below moderate to above } \\
\text { moderate and then to } \\
\text { below moderate }\end{array}$ & $\begin{array}{l}\text { Moderate to moderately } \\
\text { high and then to } \\
\text { moderate }\end{array}$ & 15.8 & 45.6 \\
\hline 6 & 29 & 13.3 & High to zero earlier & High to zero & 4.1 & 44.9 \\
\hline 7 & 16 & 7.3 & Moderate to low & Moderate to low & 2.7 & 5.8 \\
\hline Total & 218 & 100 & & & & \\
\hline
\end{tabular}

associated plots of Figure 2 since these latter plots are based on averages.

\section{Risk factors for hospitalization}

The adaptively generated dichotomous risk factor based on the seven adherence types for predicting hospitalization was the poor-adherence type, consisting of clusters 4,6 , and 7 , versus better adherence, consisting of clusters $1-3$ and 5 . Table 8 presents results for characterizing hospitalization, considering the variables of Tables 1-6. Individual risk-factor analyses identified 21 significant $(P<0.05)$ risk factors for hospitalization: two demographic, zero social support, seven clinical, five self-care, six symptom, and one cognition. The cutoff for a substantial percentage decrease in LCV scores for a sample of size 218 is $0.68 \%$. The percentage decrease exceeded this cutoff for $16(76.2 \%)$ of the 21 variables (LCV scores not reported), indicating that $\mathrm{LCV}$ ratio tests are more conservative than

Table 8 Significant individual risk factors for hospitalization

\begin{tabular}{|c|c|c|c|c|c|c|}
\hline Variable type & Variable & Risk factor & At-risk group, n (\%) & $P$-value & OR & $95 \% \mathrm{Cl}$ \\
\hline \multirow[t]{2}{*}{ Demographic } & Age, years & $\geq 74$ vs $<74$ & $41(18.8)$ & 0.029 & 2.15 & $1.08-4.29$ \\
\hline & ANART score & $\leq 34$ vs $>34$ & $123(56.4)$ & 0.017 & 2.01 & $1.13-3.57$ \\
\hline Social support & - & & & & & \\
\hline \multirow[t]{7}{*}{ Clinical } & Body mass index & $\geq 4$ I vs $<4$ I & $27(12.4)$ & 0.029 & 2.48 & $1.10-5.6 \mid$ \\
\hline & Blood urea nitrogen ${ }^{\mathrm{b}}$ & $\geq 21$ vs $<21$ or missing & $115(52.8)$ & 0.040 & 1.81 & $1.03-3.17$ \\
\hline & Charlson total & $\geq 3$ vs $<3$ & $100(45.9)$ & 0.006 & 2.19 & $1.25-3.85$ \\
\hline & Comorbidities & $\geq 2$ vs $<2$ & I6I (73.9) & 0.016 & 2.35 & I.|8-4.7| \\
\hline & Creatinine & $\geq 0.98$ vs $<0.98$ or missing & $146(67.0)$ & 0.035 & 1.95 & $1.05-3.62$ \\
\hline & Ejection fraction & $\leq 30$ vs $>30$ or missing & $105(48.2)$ & 0.026 & 1.89 & $|.08-3.3|$ \\
\hline & Hemoglobin & $\leq 14.1$ vs $>14.1$ or missing & $149(68.3)$ & 0.003 & 2.71 & $1.40-5.23$ \\
\hline \multirow[t]{5}{*}{ Self-care } & Adherence type & Poor vs better & $63(28.9)$ & $<0.00 \mathrm{I}$ & 3.47 & $1.89-6.39$ \\
\hline & SCHFI self-care management & $\leq 92$ vs $>92$ or missing ${ }^{c}$ & $89(40.8)$ & 0.027 & 1.88 & $1.07-3.30$ \\
\hline & Total medications & $\geq 12$ vs $<12$ & 71 (32.6) & $<0.00 \mathrm{I}$ & 3.25 & $1.80-5.89$ \\
\hline & Consistency, \% & $\leq \mathrm{I}$ vs $>\mathrm{I}$ & $29(13.3)$ & 0.001 & 3.61 & $1.76-7.40$ \\
\hline & Prescribed doses taken, $\%$ & $\leq 44$ vs $>44$ & $38(17.4)$ & $<0.00 \mathrm{I}$ & 3.90 & $1.88-8.10$ \\
\hline \multirow[t]{6}{*}{ Symptom } & $\begin{array}{l}\text { Trouble breathing or ankle } \\
\text { swelling within past month }\end{array}$ & Yes vs no & $94(43.1)$ & 0.025 & 1.90 & $1.09-3.32$ \\
\hline & General health perception & Poor vs fair to excellent & $24(11.0)$ & 0.002 & 4.16 & $1.69-10.23$ \\
\hline & Health compared to a year ago & $\begin{array}{l}\text { Much worse now than } \\
\text { a year ago vs not much } \\
\text { worse now than a year ago }\end{array}$ & $130(59.6)$ & 0.049 & 1.79 & $1.00-3.20$ \\
\hline & NYHA class & IV vs I-III & $41(18.8)$ & 0.001 & 3.13 & $1.56-6.29$ \\
\hline & Fatigue & $\geq 6$ vs $<6$ & $132(60.6)$ & 0.009 & 2.21 & $1.22-4.01$ \\
\hline & PHQ total & $\geq 12$ vs $<12$ & $12(5.5)$ & 0.034 & 3.80 & $1.11-13.1$ \\
\hline Cognition & TICS score & $\leq 29$ vs $>29$ & $36(16.5)$ & 0.014 & 3.26 & $1.27-8.17$ \\
\hline
\end{tabular}

Notes: a ${ }^{\circ}$ ut of 218 patients with some Medication Event Monitoring System data; b the adaptive classification is equivalent to the standard classification of $>20$ vs $\leq 20$; 'patients with missing SCHFI self care management score were the ones who did not have trouble breathing or ankle swelling.

Abbreviations: OR, odds ratio; Cl, confidence interval; ANART, American National Adult Reading Test; NYHA, New York Heart Association; PHQ, Patient Health Questionnaire; SCHFI, Self-Care of Heart Failure Index; TICS, Telephone Interview for Cognitive Status; vs, versus. 
Table 9 Multiple risk-factor model for hospitalization

\begin{tabular}{|c|c|c|c|c|c|c|}
\hline Variable type & Variable & Risk factor & At-risk group, $\mathrm{n}(\%)^{\mathrm{a}}$ & $P$-value & OR & $95 \% \mathrm{Cl}$ \\
\hline Demographic & - & & & & & \\
\hline Social support & - & & & & & \\
\hline \multirow[t]{2}{*}{ Clinical } & Hemoglobin & $\leq \mid 4.1$ vs $>14.1$ or missing & $149(68.3)$ & 0.008 & 2.74 & $1.30-5.80$ \\
\hline & Ejection fraction & $\leq 30$ vs $>30$ or missing & $105(48.2)$ & 0.010 & 2.39 & $1.24-4.62$ \\
\hline \multirow[t]{2}{*}{ Self-care } & Adherence type & Poor vs better & $63(28.9)$ & $<0.001$ & 3.41 & $1.74-6.68$ \\
\hline & Total medications & $\geq 12$ vs $<12$ & 7I (32.6) & 0.003 & 2.71 & $\mathrm{I} .4 \mathrm{I}-5.20$ \\
\hline Symptom & NYHA class & IV vs I-III & $41(18.8)$ & 0.005 & 3.22 & $1.43-7.22$ \\
\hline Cognition & - & & & & & \\
\hline
\end{tabular}

Note: ${ }^{a}$ Out of 218 patients with some Medication Event Monitoring System data.

Abbreviations: OR, odds ratio; $\mathrm{Cl}$, confidence interval; NYHA, New York Heart Association; vs, versus.

tests for zero coefficients, and so are similar to multiplecomparison approaches. All standard classifications except the one for BUN were nonsignificant, but this was equivalent to the adaptive BUN classification.

Results for the adaptive multiple risk-factor model generated considering the 21 significant risk factors of Table 8 are reported in Table 9. This model had five risk factors: lower Hgb, lower ejection fraction, poor adherence type, higher total number of prescribed medications, and NYHA class IV. The model had two clinical, two self-care, and one symptom risk factors, and no demographic, social support, or cognition risk factors. The LCV score was 0.56979 . In contrast, the best individual risk-factor model based on poor MEMS adherence had an LCV score of 0.53527 , with substantial percentage decrease of $6.06 \%$, indicating that the multiple risk-factor model substantially improved on each of the individual risk-factor models.

Interactions with poor-adherence type were identified for three risk factors: poor-adherence type with lower ANART score ( $\leq 34,32$ [14.7\%] patients), with higher MSPSS score ( $\geq 54,59$ [27.1\%] patients), and with lower LNS score $(\leq 8,41[18.8 \%]$ patients). The percentage decrease in LCV score for the model based on the poor-adherence type was insubstantial at $0.35 \%$ for the second of these interactions (with MSPSS score), but was substantial at $1.35 \%$ for the first and at $1.68 \%$ for the third.

The adaptive multiple risk-factor model generated considering these three interactions and the 21 significant risk factors of Table 8 included the single interaction between the poor-adherence type and lower LNS score. It also included the same four nonadherence risk factors from the noninteraction model of Table 9. The LCV score was 0.58318 , which was a substantial improvement on the noninteraction multiple risk-factor model with percentage decrease in the LCV score of $2.30 \%$.

The adaptive model considering pair-wise interactions is described in Table 10 and included seven interactions (and no noninteraction risk factors):

1. poor-adherence type and lower LNS score

2. higher number of comorbid conditions and higher number of medications

3. higher BUN and lower percentage PDT

Table 10 Multiple risk-factor interaction model for hospitalization

\begin{tabular}{|c|c|c|c|c|c|c|c|}
\hline \multicolumn{2}{|c|}{ Interaction term I } & \multicolumn{2}{|c|}{ Interaction term 2} & \multirow{2}{*}{$\begin{array}{l}\text { At-risk group, } \\
\text { n (\%) }\end{array}$} & \multirow[t]{2}{*}{$P$-value } & \multirow[t]{2}{*}{ OR } & \multirow[t]{2}{*}{$95 \% \mathrm{Cl}$} \\
\hline Variable & Risk factor & Variable & Risk factor & & & & \\
\hline Adherence type & Poor vs better & LNS score & $\leq 8$ vs $>8$ or missing & $32(14.7)$ & $<0.001$ & 6.40 & $2.30-17.8$ \\
\hline Comorbidities & $\geq 2$ vs $<2$ & Total medications & $\geq 12$ vs $<12$ & $64(29.4)$ & 0.001 & 3.46 & I.68-7.I I \\
\hline $\begin{array}{l}\text { Blood urea } \\
\text { nitrogen }\end{array}$ & $\geq 2$ I vs $<2$ I & $\begin{array}{l}\text { Prescribed doses } \\
\text { taken, \% }\end{array}$ & $\leq 44$ vs $>44$ & $21(9.6)$ & 0.035 & 4.29 & $1.14-16.2$ \\
\hline Hemoglobin & $\begin{array}{l}\leq 14.1 \text { vs }>14.1 \\
\text { or missing }\end{array}$ & $\begin{array}{l}\text { Health compared } \\
\text { to a year ago }\end{array}$ & $\begin{array}{l}\text { Much worse now } \\
\text { than a year ago vs } \\
\text { not much worse } \\
\text { now than a year ago }\end{array}$ & $89(40.8)$ & 0.009 & 2.51 & $1.26-4.99$ \\
\hline Age & $\geq 74$ vs $<74$ & TICS score & $\leq 29$ vs $>29$ & II (5.0) & 0.008 & 11.70 & $1.92-7 \mid .7$ \\
\hline Body mass index & $\geq 4$ I vs $<4$ I or missing & Hemoglobin & $\leq 14.1$ vs $>14.1$ or missing & $23(10.6)$ & 0.003 & 5.08 & $1.77-14.6$ \\
\hline Ejection fraction & $\leq 30$ vs $>30$ or missing & Fatigue & $\geq 6$ vs $<6$ & $62(28.4)$ & 0.008 & 2.80 & $1.31-5.96$ \\
\hline
\end{tabular}

Note: a ${ }^{O}$ ut of 218 patients with some Medication Event Monitoring System data.

Abbreviations: OR, odds ratio; Cl, confidence interval; LNS, Letter-Number Sequencing; TICS, Telephone Interview for Cognitive Status; vs, versus. 
4. lower Hgb and much worse perceived health now compared to last year

5. older age and lower TICS score

6. higher BMI and lower Hgb

7. lower ejection fraction and higher fatigue.

The $C$-index for this model was 0.83 (values between 0.8 to 0.9 are considered to provide excellent discrimination ${ }^{39}$ ).

To assess the possibility of collinearity between these seven interactions, we computed logistic regression models predicting each of these seven interactions as a function of the other six. The largest Nagelkerke $R^{2}$ value for these models was $9.5 \%$, indicating that collinearity was not a problem for the seven-interaction model.

Patients had zero to five of these risk factors. Of the 62, $63,59,19$, eleven, and four patients with zero to five of these interaction risk factors, respectively, the percentage hospitalized was $9.7 \%, 19.1 \%, 54.2 \%, 79.0 \%, 90.9 \%$, and $100 \%$. The hospitalization risk index model based on the count of the number of these risk factors as the only predictor of hospitalization had an LCV score of 0.61594, which provided a substantial improvement over the seven-interaction model, with percentage decrease in the LCV score of $1.18 \%$. This model has a $C$-index of 0.82 . As an example, using a cutoff of 0.5 for generating predictions (ie, predictions based on maximum likelihood), the sensitivity is $77.2 \%$ and the specificity $77.0 \%$, and so well balanced.

\section{Discussion}

In this prospective study, we confirmed that both a poor type of medication adherence pattern and a low percentage of prescribed doses taken were important predictors of hospitalization in adults with HF. In addition, we identified low $\mathrm{Hgb}$, depressed ejection fraction, NYHA class IV, and taking 12 or more medications daily as risk factors for hospitalization. When interactions were considered, seven combinations of factors increased the predictive capability of the model: 1) poor-adherence type and lower score on a test of shortterm memory, 2) higher number of comorbid conditions and higher number of daily medications, 3 ) poor kidney function (higher BUN) and lower percentage prescribed doses taken, 4) lower Hgb and perceptions of poor health, 5) older age and lower score on the TICS, 6) higher BMI and lower Hgb, and 7) lower ejection fraction and higher fatigue. Patients with none of these seven interactions were unlikely to be hospitalized, but all of those with five of the seven interaction risk factors were hospitalized over the 6-month period.

A pattern of poor medication adherence and taking a low percentage of prescribed doses were both associated with hospitalization in this sample. These results are consistent with prior research, ${ }^{13,14,40,41}$ but those studies measured adherence using administrative claims data or retrospective review of hospital records, so the pattern of adherence and the percentage of doses taken could only be inferred. What this study adds is the prospective analysis of individual patterns of medication-taking behavior in relation to hospitalization.

An important factor interacting with a poor medicationadherence type was impaired cognition. Cognitive deficits occur in a significant proportion of adults with chronic $\mathrm{HF},{ }^{42}$ including deficits in memory. ${ }^{43}$ Prospective memory has been demonstrated to be related to medication adherence. ${ }^{44}$ Although short-term memory as measured by the LNS and prospective memory are related, prospective memory is more encompassing, referring specifically to the ability to remember to do something in the future. ${ }^{44}$ As taking medication on a schedule requires that patients remember to do so, this result suggests that HF patients who have not compensated for poor memory with a strategy to assist them to remember medications are at increased risk of hospitalization. One physiologic factor known to be associated with impaired cognition is poor kidney function. ${ }^{45}$ In this study, higher BUN levels interacted with taking a lower percentage of prescribed medication doses to predict hospitalization. A memory device to facilitate adherence may be particularly important in HF patients with impaired kidney function.

We found that patients taking multiple daily medications were likely to be hospitalized, perhaps because they were not taking their medications as prescribed. ${ }^{46}$ This was especially true in patients with more comorbid illnesses, where the number of medications and comorbid illnesses interacted to predict hospitalizations. This result is not surprising, considering the profile of those individuals with multiple chronic conditions who are taking numerous medications. Illness symptoms and medication side effects can be expected to make routine medication adherence challenging. ${ }^{47}$

Low $\mathrm{Hgb}$, low ejection fraction, and poor functional status all predicted hospitalization. Previous investigators have identified a wide variety of clinical factors associated with hospitalization, including anemia. ${ }^{48}$ Although correction of anemia has been shown to slow the progression of $\mathrm{HF}$ and reduce hospitalization rates, ${ }^{49}$ more than one-third of the participants in our study were anemic using the World Health Organization definitions of $<13 \%$ for men and $<12 \%$ for women. Correction of anemia with subcutaneous erythropoietin is advocated in clinical guidelines and covered in the US by Medicare, ${ }^{50}$ but expense still may be limiting its widespread use. Low Hgb interacted with self-reported 
perceptions of poor health, suggesting that those patients who were anemic were symptomatic enough to label themselves as getting worse over time. Low Hgb also interacted with high BMI, as might be expected for two conditions that cause high output failure. Depressed ejection fraction and poor NYHA functional class were both independently associated with hospitalization, as has been shown by other investigators. $^{2}$

Limitations of this study include the relatively small sample size, compared to epidemiologic studies conducted with thousands of respondents, collected from a single region in the US. Participants were younger and better educated than other community samples. Strengths include the prospective design, the objective measurement of medication adherence, and the sophisticated statistical approach that accounted for general nonlinear adherence trajectories rather than simply categorizing adherence using the $>80 \%$ cutoff in percentage PDT used by most prior investigators. This nuanced approach to understanding medication-adherence patterns provides important insights into patient behavior that cannot be discerned from epidemiologic research.

Future research is needed to identify predictors of nonadherence. The World Health Organization adherence model suggests that socioeconomic, condition, therapy, patient, and health care-system dimensions contribute to nonadherence, ${ }^{51}$ and further research is needed to explore these dimensions. Efforts to identify the strategies used by HF patients to remember medications is needed. Finally, it will be important to include cost in future studies, because Sokol et al ${ }^{40}$ demonstrated that although nonadherence was associated with higher hospitalization risk in HF patients, there were no differences in costs compared to those who were more adherent.

\section{Acknowledgments}

This work was funded by a grant from the National Heart, Lung and Blood Institute (R01 HL084394-01A1) and by the Philadelphia Veterans Affairs Medical Center, VISN 4 Mental Illness Research, Education, and Clinical Center (MIREC). The authors gratefully acknowledge Megan Patey, RN, BSN for her assistance with preparation of the data for analysis.

\section{Disclosure}

The authors report no conflicts of interest in this work.

\section{References}

1. Heidenreich PA, Albert NM, Allen LA, et al. Forecasting the Impact of Heart Failure in the United States: a policy statement From the American Heart Association. Circ Heart Failure. 2013;6(3):606-619.
2. Giamouzis G, Kalogeropoulos A, Georgiopoulou V, et al. Hospitalization epidemic in patients with heart failure: risk factors, risk prediction, knowledge gaps, and future directions. J Card Fail. 2011;17(1): 54-75.

3. Philbin EF, DiSalvo TG. Prediction of hospital readmission for heart failure: development of a simple risk score based on administrative data. J Am Coll Cardiol. 1999;33(6):1560-1566.

4. Krumholz HM, Chen YT, Wang Y, Vaccarino V, Radford MJ, Horwitz RI. Predictors of readmission among elderly survivors of admission with heart failure. Am Heart J. 2000;139(1 Pt 1):72-77.

5. Felker GM, Leimberger JD, Califf RM, et al. Risk stratification after hospitalization for decompensated heart failure. J Card Fail. 2004; 10(6):460-466.

6. Pocock SJ, Wang D, Pfeffer MA, et al. Predictors of mortality and morbidity in patients with chronic heart failure. Eur Heart J. 2006; 27(1):65-75.

7. Yamokoski LM, Hasselblad V, Moser DK, et al. Prediction of rehospitalization and death in severe heart failure by physicians and nurses of the ESCAPE trial. $J$ Card Fail. 2007;13(1):8-13.

8. Keenan PS, Normand SL, Lin Z, et al. An administrative claims measure suitable for profiling hospital performance on the basis of 30-day all-cause readmission rates among patients with heart failure. Circ Cardiovasc Qual Outcomes. 2008;1(1):29-37.

9. Au AG, McAlister FA, Bakal JA, Ezekowitz J, Kaul P, van Walraven C. Predicting the risk of unplanned readmission or death within 30 days of discharge after a heart failure hospitalization. Am Heart J. 2012;164(3):365-372.

10. Coleman CI, Limone B, Sobieraj DM, et al. Dosing frequency and medication adherence in chronic disease. J Manage Care Pharm. 2012;18(7):527-539.

11. Wu JR, Moser DK, Lennie TA, Burkhart PV. Medication adherence in patients who have heart failure: a review of the literature. Nurs Clin North Am. 2008;43(1):133-153; vii-viii.

12. Correia J, Silva FF, Roque C, Vieira H, Providencia LA. Impact of a specialized outpatient heart failure follow-up program on hospitalization frequency and functional status of patients with advanced heart failure. Rev Port Cardiol. 2007;26(4):335-343.

13. Esposito D, Bagchi AD, Verdier JM, Bencio DS, Kim MS. Medicaid beneficiaries with congestive heart failure: association of medication adherence with healthcare use and costs. Am J Manag Care. 2009; 15(7):437-445.

14. Fitzgerald AA, Powers JD, Ho PM, et al. Impact of medication nonadherence on hospitalizations and mortality in heart failure. J Card Fail. 2011;17(8):664-669.

15. Ambardekar AV, Fonarow GC, Hernandez AF, Pan W, Yancy CW, Krantz MJ. Characteristics and in-hospital outcomes for nonadherent patients with heart failure: findings from Get With The Guidelines Heart Failure (GWTG-HF). Am Heart J. 2009;158(4):644-652.

16. Cole JA, Norman H, Weatherby LB, Walker AM. Drug copayment and adherence in chronic heart failure: effect on cost and outcomes. Pharmacotherapy. 2006;26(8):1157-1164.

17. Knafl GJ, Delucchi KL, Bova CA, Fennie KP, Ding K, Williams AB. A systematic approach for analyzing electronically monitored adherence data. In: Ekwall B, Cronquist M, editors. Micro Electro Mechanical Systems (MEMS) Technology, Fabrication Processes and Applications. Hauppauge (NY): Nova Science; 2010:1-66.

18. Knafl GJ, Fennie KP, Bova C, Dieckhaus K, Williams AB. Electronic monitoring device event modelling on an individual-subject basis using adaptive Poisson regression. Stat Med. 2004;23(5):783-801.

19. Wu JR, Moser DK, De Jong MJ, et al. Defining an evidence-based cutpoint for medication adherence in heart failure. Am Heart $J$. 2009;157(2):285-291.

20. Bova C, Fennie KP, Dieckhaus K, Knafl GJ, Watrous E, Williams AB. Use of electronic monitoring devices to measure antiretroviral adherence: practical considerations. AIDS Behav. 2005;9(1):103-110.

21. Knafl GJ, Schoenthaler A, Ogedegbe G. Secondary analysis of electronically monitored medication adherence data for a cohort of hypertensive African-Americans. Patient Prefer Adherence. 2012;6: 207-219. 
22. Riegel B, Moelter ST, Ratcliffe SJ, et al. Excessive daytime sleepiness is associated with poor medication adherence in adults with heart failure. $J$ Card Fail. 2011;17(4):340-348.

23. Buckwalter JG, Crooks VC, Petitti DB. A preliminary psychometric analysis of a computer-assisted administration of the Telephone Interview of Cognitive Status - modified. J Clin Exp Neuropsychol. 2002;24(2):168-175.

24. Riegel B, Lee CS, Ratcliffe SJ, et al. Predictors of objectively measured medication nonadherence in adults with heart failure. Circ Heart Fail. 2012;5(4):430-436.

25. Zimet GD, Dahlem NW, Zimet SG, Farley GK. Multidimensional scale of perceived social support. In: Fischer J, Corcoran, K, editors. Measures for Clinical Practice: A Sourcebook. Volume 2 -Adults. 2nd ed. New York: Free Press; 1994.

26. Charlson M, Pompei P, Ales K, MacKenzie C. A new method of classifying prognostic comorbidity in longitudinal studies: development and validation. J Chronic Dis. 1987;40(5):373-383.

27. Heart Failure Society of America. HFSA 2006 Comprehensive Heart Failure Practice Guideline. J Card Fail. 2006;12(1):e1-e2.

28. Riegel B, Lee CS, Dickson VV, Carlson B. An update on the self-care of heart failure index. J Cardiovasc Nurs. 2009;24(6):485-497.

29. van der Wal MH, Jaarsma T, Moser DK, van Veldhuisen DJ. Development and testing of the Dutch Heart Failure Knowledge Scale. Eur J Cardiovasc Nurs. 2005;4(4):273-277.

30. Green CP, Porter CB, Bresnahan DR, Spertus JA. Development and evaluation of the Kansas City Cardiomyopathy Questionnaire: a new health status measure for heart failure. J Am Coll Cardiol. 2000;35(5): 1245-1255.

31. MacLean AW, Fekken GC, Saskin P, Knowles JB. Psychometric evaluation of the Stanford Sleepiness Scale. J Sleep Res. 1992;1(1): 35-39.

32. Johns MW. Reliability and factor analysis of the Epworth Sleepiness Scale. Sleep. 1992;15(4):376-381.

33. Buysse D, Reynolds CF 3rd, Monk TH, Berman SR, Kupfer, DJ. The Pittsburgh Sleep Quality Index: a new instrument for psychiatric practice and research. Psychiatry Res. 1989;28(2):193-213.

34. Kroenke K, Spitzer R, Williams J. The PHQ-9: Validity of a brief depression severity measure. J Gen Intern Med. 2001;16(9): 606-613.

35. Lezak M, Howieson D, Lorig D. Neuropsychological Assessment. 4th ed. New York: Oxford University Press; 2004.

36. Dinges D, Powell J. Microcomputer analyses of performance on a portable, simple visual RT task during sustained operations. Behav Res Methods Instrum Comput. 1985;17(6):652-655.

37. McCullagh P, Nelder JB. Generalized Linear Models. Boca Raton (FL): Chapman and Hall/CRC; 1999.
38. Royston P, Altman DG. Regression using fractional polynomials of continuous covariates: parsimonious parametric modeling. Appl Stat. 1994:43(3):429-467.

39. Hosmer DW, Lemeshow S. Applied Logistic Regression. 2nd ed. New York: John Wiley \& Sons; 2000.

40. Sokol MC, McGuigan KA, Verbrugge RR, Epstein RS. Impact of medication adherence on hospitalization risk and healthcare cost. Med Care. 2005;43(6):521-530.

41. Michalsen A, Konig G, Thimme W. Preventable causative factors leading to hospital admission with decompensated heart failure. Heart. 1998;80(5):437-441.

42. Pressler SJ. Cognitive functioning and chronic heart failure: a review of the literature (2002-July 2007). J Cardiovasc Nurs. 2008;23(3):239-249.

43. Pressler SJ, Subramanian U, Kareken D, et al. Cognitive deficits in chronic heart failure. Nurs Res. 2010;59(2):127-139.

44. Zogg JB, Woods SP, Sauceda JA, Wiebe JS, Simoni JM. The role of prospective memory in medication adherence: a review of an emerging literature. J Behav Med. 2012;35(1):47-62.

45. Hailpern SM, Melamed ML, Cohen HW, Hostetter TH. Moderate chronic kidney disease and cognitive function in adults 20 to 59 years of age: Third National Health and Nutrition Examination Survey (NHANES III). J Am Soc Nephrol. 2007;18(7):2205-2213.

46. Tsai KT, Chen JH, Wen CJ, et al. Medication adherence among geriatric outpatients prescribed multiple medications. Am J Geriatr Pharmacother. 2012;10(1):61-68.

47. Dickson VV, Buck H, Riegel B. Multiple comorbid conditions challenge heart failure self-care by decreasing self-efficacy. Nurs Res. 2013;62(1):2-9.

48. Pitt B. Male gender, diabetes, COPD, anemia, and creatinine clearance $<30 \mathrm{~mL} / \mathrm{min}$ predicted hospitalization after heart failure diagnosis. Ann Intern Med. 2010;152(8):JC4-2, JC4-3.

49. Silverberg DS, Wexler D, Blum M, et al. The correction of anemia in severe resistant heart failure with erythropoietin and intravenous iron prevents the progression of both the heart and the renal failure and markedly reduces hospitalization. Clin Nephrol. 2002;58 Suppl 1: S37-S45.

50. Naci H, de Lissovoy G, Hollenbeak C, et al. Historical clinical and economic consequences of anemia management in patients with endstage renal disease on dialysis using erythropoietin stimulating agents versus routine blood transfusions: a retrospective cost-effectiveness analysis. J Med Econ. 2012;15(2):293-304.

51. World Health Organization. Adherence to long-term therapies: Evidence for action. Geneva: World Health Organization; 2003. Available from: http://www.who.int/chp/knowledge/publications/adherence_full_report. pdf. Accessed November 22, 2013.
Patient Preference and Adherence

\section{Publish your work in this journal}

Patient Preference and Adherence is an international, peer-reviewed, open access journal focusing on the growing importance of patient preference and adherence throughout the therapeutic continuum. Patient satisfaction, acceptability, quality of life, compliance, persistence and their role in developing new therapeutic modalities and compounds to

\section{Dovepress}

optimize clinical outcomes for existing disease states are major areas of interest. This journal has been accepted for indexing on PubMed Central. The manuscript management system is completely online and includes a very quick and fair peer-review system. Visit http://www.dovepress.com/ testimonials.php to read real quotes from published authors. 